\title{
L'évolution de l'enseignement de la pharmacie : Former des professionnels des soins aux patients efficaces
}

\author{
par Lalitha Raman-Wilms
}

$\mathrm{L}$ es changements décrits dans le Plan directeur pour la pharmacie soulignent le besoin de « [p] rocurer aux Canadiens un traitement médicamenteux aux résultats optimaux par des soins axés sur le patient ${ }^{1}$. Cette démarche requiert que les pharmaciens prennent part aux soins des patients axés sur les résultats tout en travaillant au sein d'équipes de soins de santé; les pharmaciens doivent aussi être responsables de l'utilisation sécuritaire et efficace des médicaments.

Le besoin de prendre cette responsabilité est criant pour tous les pharmaciens. En effet, près de la moitié des patients qui se rendent à une pharmacie communautaire ou une clinique connaissent un problème pharmacothérapeutique ${ }^{2}$. Les patients qui sont hospitalisés éprouvent également un nombre considérable d'événements indésirables évitables liés aux médicaments ${ }^{3}$. Le coût des médicaments est la dépense de notre système de santé qui croît le plus rapidement ${ }^{4}$. De plus, avec l'utilisation accrue des médicaments, il y a un risque encore plus grand d'événements indésirables liés aux médicaments. En outre, les personnes âgées utilisent le plus de médicaments, ce qui les expose à un risque encore plus élevé de problèmes pharmacothérapeutiques; en fait, d'ici 2036, un Canadien sur 4 aura plus de 65 ans ${ }^{5}$. Les pharmaciens devront donc prendre en charge efficacement la pharmacothérapie de tous les patients et les facultés de pharmacie ainsi que la profession dans son ensemble ont toutes deux des obligations de préparer les nouveaux pharmaciens à ce rôle.

L'enseignement visant à préparer les pharmaciens aux rôles élargis en matière de soins aux patients a déjà amorcé un virage. Par exemple, toutes les écoles de pharmacie au Canada se sont engagées à mettre en place un programme de doctorat professionnel en pharmacie (Pharm. D.) de premier cycle d'ici $2020^{6}$. Certains éléments clés des nouveaux programmes comprennent une approche élargie et intégrée de l'enseignement de la pharmacothérapie, des cours de gestion comportant le développement de services de soins aux patients, l'incorporation de l'examen physique et de compétences liées à l'élargissement du champ de pratique (p.ex., formation pour l'administration d'injections) ainsi qu'une formation clinique exhaustive en soins directs aux patients. Cet enseignement élargi est appuyé à l'échelle nationale par le document sur les résultats d'apprentissage publié en 2010 par l'Association des facultés de pharmacie du Canada $^{7}$ qui exigent des diplômés en pharmacie qu'ils soient des " experts en pharmacothérapie ». De plus, le Conseil canadien de l'agrément des programmes de pharmacie (CCAPP) a maintenant approuvé de nouvelles normes relatives aux programmes de Pharm. D. de premier cycle, qui seront mises en œuvre au début de l'année 2013. Chaque programme doit proposer un total de 40 semaines (1 600 heures) d'expérience pratique, dont au moins huit semaines (320 heures) en début et en milieu de programme et au moins 24 semaines (960 heures) à la fin du programme. Ce niveau de formation clinique est considéré comme essentiel pour que les diplômés deviennent des prestataires de soins aux patients autonomes et compétents, et qui sont responsables à la fois envers les patients et leurs collègues au sein des équipes interprofessionnelles.

La formation actuelle, bien qu' efficace, est exigeante pour le précepteur et le milieu de stage et ne tient pas explicitement l'étudiant responsable envers le patient et le reste de l'équipe de soins de santé. Les défis sont aussi nombreux que les occasions à l'aube de ce changement de paradigme dans la façon de former les étudiants en pharmacie.

Lors de la réunion de l'American Society of Health-System Pharmacists en juin 2011, Ashby ${ }^{8}$ a proposé un modèle de formation des étudiants en pharmacie qui permettrait aux étudiants en formation de devenir davantage responsables et de les rendre indispensables au sein de l'équipe soignante. Il a également souligné l'importance pour les étudiants d'entreprendre des activités de soins aux patients reconnues pour améliorer les résultats cliniques. Les autres recommandations étaient notamment de prolonger la durée des stages et de donner du soutien aux étudiants dans leur cheminement de carrière individuel en appariant leur formation à leurs champs d'intérêt.

Dans ce numéro du JCPH, Hall et ses collaborateurs ${ }^{9}$ présentent un survol informatif de la formation expérientielle en pharmacie hospitalière et proposent huit principes directeurs 
qu'ils considèrent comme des facteurs clés de la réussite des futurs modèles de formation expérientielle. Beaucoup de ce qu'ils présentent s'apparente à la formation dans les programmes de médecine, notamment amorcer la formation expérientielle plus tôt dans le programme; fournir tôt un tandem d'expérience en milieux hospitalier et communautaire, avec une pratique plus soutenue les dernières années (p. ex., le modèle de pratique pharmaceutique avancée longitudinale ou Longitudinal Advanced Pharmacy Practice [LAPP]); incorporer un programme d'appariement pour les stages de type LAPP; incorporer des modèles d'apprentissage par les pairs ou les quasi-pairs; inclure de la formation interdisciplinaire; et faire participer les étudiants à des activités qui entraîneront des résultats positifs pour les patients. En dernier lieu, les auteurs suggèrent que la Société canadienne des pharmaciens d'hôpitaux travaille avec les praticiens et les facultés de pharmacie afin de développer les meilleures pratiques pour la formation des étudiants.

Ce sont tous des éléments importants à considérer qui, une fois en place, pourraient rendre les diplômés fin prêts à prodiguer des soins directs aux patients. Cela demande aussi que les étudiants deviennent des membres importants et actifs de l'équipe de soins de santé et soient responsables de leurs patients.

Globalement, la profession et les établissements d'enseignement appuieront vraisemblablement les principes décrits par Hall et ses collaborateurs?. Mais il y aura des défis. La formation des étudiants en pharmacie n'est pas financée par le gouvernement dans la même mesure que les autres disciplines de la santé. Nous devons également changer la culture organisationnelle des facultés de pharmacie et la mentalité des précepteurs, pour qu'ils soient prêts et disposés à amorcer ce virage significatif dans l'administration de la formation. Un tel changement de culture pourrait signifier qu'il faut permettre aux étudiants d'apprendre de leurs erreurs dès le début, tout en s'assurant que les soins aux patients ne sont pas compromis. Nous devrons élargir le bassin de précepteurs et donner du soutien au perfectionnement des précepteurs. Finalement, les pharmaciens hospitaliers seront appelés à travailler plus étroitement avec les établissements d'enseignement qui sont en définitive responsables de la mise en œuvre des programmes, pour veiller à ce que les diplômés acquièrent les compétences requises.

Le projet du Plan directeur pour la pharmacie a déjà commencé à faciliter des discussions pour répondre à certaines de ces questions à l'échelle nationale. En outre, des facultés de pharmacie ont commencé à solliciter le point de vue de partenaires hospitaliers locaux sur les questions relatives à la formation des étudiants. Les facultés et la profession doivent évaluer les services que fournissent les étudiants afin d'en démontrer la portée à la fois sur les résultats cliniques et sur la capacité des établissements à bonifier la prestation des services du pharmacien. Des mécanismes doivent aussi être mis de l'avant pour favoriser des partenariats avec d'autres systèmes de santé et établissements communautaires afin qu'ils participent aux nouveaux modèles de formation des étudiants en pharma- cie. La mise sur pied de " communautés de praticiens " proposant diverses formations aux étudiants pourrait favoriser un meilleur partage des ressources. Au bout du compte, nous devons travailler avec le gouvernement pour faire valoir la nécessité d'un soutien financier à la formation des étudiants en pharmacie.

Les établissements d'enseignement, les pharmacies hospitalières et communautaires ainsi que les précepteurs doivent évaluer le meilleur moyen d'adapter les principes directeurs soulignés par Hall et ses collaborateurs?. Tout comme il est urgent qu'un plus grand nombre de pharmaciens assument les responsabilités des soins directs aux patients, les établissements, les partenaires communautaires et le milieu universitaire doivent sans délai travailler ensemble à la réalisation de cette vision commune pour que les futurs diplômés deviennent des prestataires efficaces de soins directs aux patients.

[Traduction par l'éditeur]

\section{References}

1. Plan directeur pour la pharmacie : La vision de la pharmacie. Ottawa $(\mathrm{ON})$ : Association des pharmaciens du Canada; 2012. Publié au www. pharmacists.ca/index.cfm/pharmacy-in-canada/blueprint-for-pharmacy/. Consulté le 27 mai 2012.

2. Cipolle RJ, Strand LM, Morley PC. Pharmaceutical care practice: the clinician's guide. 2nd ed. New York (NY) : McGraw-Hill Companies, Inc; 2004.

3. Baker GR, Norton PG, Flintoft V, Blais R, Brown A, Cox J, et al. 2004. The Canadian Adverse Events Study: the incidence of adverse events among hospital patients in Canada. CMAJ 2004;170(11):1678-1686.

4. Nigam R, Mackinnon NJ, U D, Hartnell NR, Levy AR, Gurnham ME, et al. Development of Canadian safety indicators for medication use. Healthc Q 2008;11(3 Spec No.):47-53.

5. Projections démographiques pour le Canada, les provinces et les territoires 2009 à 2036. Ottawa (ON) : Statistique Canada; 2010. Publié au http://www.statcan.gc.ca/pub/91-520-x/91-520-x2010001-fra.pdf. Consulté le 27 mai 2012.

6. Position statement and joint resolution on the Doctor of Pharmacy (Phar$\mathrm{mD}$ ) for the first professional degree at universities in Canada. Association des facultés de pharmacie du Canada et Association des doyens de pharmacie du Canada; février 2010. Publié au www.afpc.info/downloads/ 1/AFPC_ADPC_PharmD_Position_Statement_Resolution_Sept_2010.pdf. Consulté le 17 mai 2012.

7. Educational outcomes for first professional degree programs in pharmacy (entryto-practice pharmacy programs) in Canada. Vancouver (BC) : Association des facultés de pharmacie du Canada; 3 juin 2010. Publié au http:// afpc.info/downloads/1/AFPC_Education_Outcomes_AGM_June_2010.pdf. Consulté le 26 mai 2012.

8. Ashby DM. Permission granted. Am J Health Syst Pharm 2011;68(16): $1497-1504$.

9. Hall K, Musing E, Miller DA, Tisdale JE. Experiential training for pharmacy students: time for a new approach. Can J Hosp Pharm 2012;65(4):285-293.

Lalitha Raman-Wilms, B. Sc. Pharm., Pharm. D., FCSHP est professeure agrégée et vice-doyenne, Programmes professionnels, Faculté de pharmacie Leslie Dan de l'Université de Toronto à Toronto, en Ontario. Elle est également rédactrice adjointe du JCPH et membre du conseil d'administration du Conseil canadien de l'agrément des programmes de pharmacie.

\section{Address correspondence to:}

D' Lalitha Raman-Wilms

Leslie Dan Faculty of Pharmacy

University of Toronto

144 College Street

Toronto ON M5S 3M2

Courriel : I.raman.wilms.a@utoronto.ca 